\title{
Aplicação sequencial de glyphosate em plantas aquáticas de difícil controle em condição de mesocosmo
}

\section{Sequential application of glyphosate in aquatic plant of difficult control in mesocosm condition}

\author{
Claudinei da Cruz ${ }^{1}$; João Henrique Corti Cervoni ${ }^{1}$; Adilson Ferreira Silva ${ }^{2}$; Nathalia Garlich²; \\ Robinson Antonio Pitelli \\ ${ }^{1}$ Laboratório de Ecotoxicologia e Eficácia dos Agrotóxicos, LEEA, do Centro Universitário da Fundação \\ Educacional de Barretos. E-mail: claudineicruz@gmail.com, Tel. 0xx17-3321-6411 \\ ${ }^{2}$ Programa de Pós-graduação em Agronomia - Produção Vegetal da Unesp, Jaboticabal. \\ ${ }^{3}$ Ecosafe Agricultura e Meio Ambiente.
}

\section{Resumo}

O objetivo deste estudo foi avaliar a eficácia do herbicida glyphosate no controle de Salvinia herzogii, S. molesta e S. oblongifolia em aplicação sequencial. O experimento foi desenvolvido em 12 mesocosmos com capacidade de 1.080 litros. Após a colonização de $100 \%$ pelas plantas, foi realizada a primeira aplicação de glyphosate, nas doses de $2.880,0,3.360,0$ e 3840,0 g i.a. ha ${ }^{-1}$, com adição de $0,5 \%$ do adjuvante Aterbane ${ }^{\circledR}$ BR e um controle para cada espécie, com 3 repetições por tratamento. Aos 21 dias após a primeira aplicação (DAPA) foi realizada a secunda aplicação de glyphosate na dose $2.880,0 \mathrm{~g} \mathrm{ha}^{-1}$ em todos os tratamentos. Em 21 DAPA ocorreu $80 \%$ de controle em $3.840,0 \mathrm{~g} \mathrm{ha}^{-1}$ para a S. herzogii, $80 \%$ a $85 \%$ para a $S$. molesta e $90 \%$ a $93 \%$ para a S. oblongifolia em todas as doses testadas. Na fase final do experimento, aos 45 dias após a secunda aplicação (DASA) ocorreu $100 \%$ de controle para S. molesta e S. oblongifolia e para S. herzogii o controle variou de $90 \%$ a $95 \%$. A aplicação sequencial do glyphosate com o adjuvante pode ser uma alternativa para o controle de salvinias, devido ao aumento do efeito de controle por um longo período, diminuindo a capacidade de adaptação dessas plantas ao manejo químico.

Palavras chaves: método de controle, herbicidas, macrófita, Salviniaceae.

Abstract - This study aimed to evaluate the efficacy of the sequential application of glyphosate in the control of Salvinia herzogii, S. molesta and S. oblongifolia. The experiment was carried out in 12 mesocosms (1080 liters). After the the plants reached 100\% colonization, we performed the first application of glyphosate in the doses of $2880.0,3360.0$, and 3840.0 g i.a. ha ${ }^{-1}$ plus $0.5 \%$ of the Aterbane ${ }^{\circledR}$ BR surfactant; and one control, with 3 replicates per treatment. The second application of glyphosate was performed 21 days after the first application (DAPA), at the dose of $2880.0 \mathrm{~g} \mathrm{ha}^{-1}$ in all treatments. In 21 DAPA, we observed $80 \%$ of control with $3840.0 \mathrm{~g} \mathrm{ha}^{-1}$ for $S$. herzogii, $80-85 \%$ for $S$. molesta and $90-93 \%$ for S. oblongifolia at all doses tested. In the final phase of the experiment, at 45 days after the second application (DASA), we observed $100 \%$ control for S. molesta and S. oblongifolia; and for S. herzogii the control ranged from 90 to $95 \%$. The sequential application of glyphosate with the adjuvant may be an alternative for the control of salvinias, due to the control effect over a long period, reducing the adaptability of these plants to chemical management.

Key words: control method, herbicides, macrophyte, Salviniaceae

\footnotetext{
Autor Correspondente:

Claudinei da Cruz

Laboratório de Ecotoxicologia e Eficácia dos Agrotóxicos, LEEA, do Centro Universitário da Fundação

Educacional de Barretos.
}

Tel. 0xx17-3321-6411

claudineicruz@gmail.com

Recebido em: 17/10/2017

Aceito para publicação em: 23/10/2017

https://doi.org/10.4322/1980-0029.132017 


\section{Introdução}

As plantas aquáticas se tornaram problema mundial, com interferência nos principais ecossistemas aquáticos, alterando sua dinâmica biológica ou interferindo nos usos múltiplos da água. Dentre todos os problemas descritos causados por essas plantas, o aumento da evapotranspiração da água tem se tornado uma preocupação (BORIN et al., 2011; ANDA et al., 2015). O aumento de nutrientes pode causar alterações nos corpos hídricos e favorece o desenvolvimento de colonizações monoespecíficas dessas plantas, além de prejudicar a geração de energia, navegação e captura de pescados, reduz o oxigênio dissolvido (GETTYS et al., 2014).

Dentre as plantas flutuantes, as Salvinias, que pertencem a família botânica Salviniaceae, são plantas flutuantes conhecidas popularmente como samambaias d'água, com emissão de folhas a partir do caule abaxial e são encontradas em diversos corpos hídricos, desenvolvendo-se rapidamente por propagação vegetativa ou esporos e formando enorme massa vegetativa na superfície d'água (GETTYS et al., 2014). Além disso, suas folhas exibem grande quantidade de tricomas na epiderme, o que forma uma barreira física que dificulta a passagem de líquidos (BARTHLOTT, 2009; MUDGE et al., 2016).

A maior dificuldade no controle químico dessas plantas relaciona-se à rápida multiplicação vegetativa, à grande área de tecido fotossintético e à capacidade de produção de densas esteiras com plantas em camadas, umas em cima das outras, dificultando assim que se atinja as camadas mais baixas e demandando aplicações múltiplas para obtenção de um controle a longo prazo (GETTYS et al., 2014).

A eficácia do glyphosate para plantas aquáticas foi relatada para Ludwigia peploides
(SARTAIN et al., 2015), Persicaria hydropiperoides (MADSEN e t al., 2015), E. crassipes, P. stratiotes, Salvinia herzogii e Urochloa subquadripara (CRUZ et al., 2015a) e Salvinia molesta (MUDGE et al., 2016). Nesse contexto, o glyphosate pode ser uma opção para o controle de plantas aquáticas flutuantes, em virtude de sua rápida degradação em água e das restrições mínimas quanto ao uso da água tratada (GETTYS et al., 2014). Assim, o objetivo deste estudo foi determinar a eficácia do herbicida glyphosate, acrescido de $0,5 \%$ do adjuvante Aterbane ${ }^{\circledR}$, para o controle de Salvinia oblongifolia, S. herzogii e S. molesta, em aplicação sequencial, em condição de mesocosmos.

\section{Material e métodos}

Os produtos comerciais utilizados foram o Rodeo $^{\circledR}$, com 480,0 $\mathrm{g} \mathrm{L}^{-1}$ de glyphosate (herbicida) e Aterbane ${ }^{\circledR}$ BR, com 309,0 $\mathrm{g} \mathrm{L}^{-1}$ de nonil fenol etoxilado $+141,4 \mathrm{~g} \mathrm{~L}^{-1}$ de ácido dodecilbenzeno sulfônico (adjuvante).

O experimento foi conduzido em 12 mesocosmos com capacidade de 1.080 litros, com entrada e saída da água pela superfície do sistema (figura 1). O sedimento $(10 \mathrm{~cm})$ utilizado foi uma mistura de areia, solo de barranco e composto orgânico (na proporção 1:2:1 $\mathrm{vV}^{-1}$ ). Após a deposição do sedimento, os mesocosmos foram preenchidos com água e introduzidas as três espécies de salvinias em cada mesocosmo. Depois da colonização de $100 \%$ da superfície dos mesocosmos em torno de 30 dias (figura 1), o herbicida, acrescido de $0,5 \%$ $\left(\mathrm{v} \mathrm{v}^{-1}\right)$ de adjuvante Aterbane ${ }^{\circledR}$, foi aplicado nas doses de 2.880,0; 3.360,0; e 3.840,0 g i.a. ha ${ }^{-1}$. Após 21 dias da primeira aplicação (DAPA) foi realizada a aplicação sequencial com $2.880,0 \mathrm{~g} \mathrm{ha}^{-1}$ de glyphosate, em todos os tratamentos e mantidos

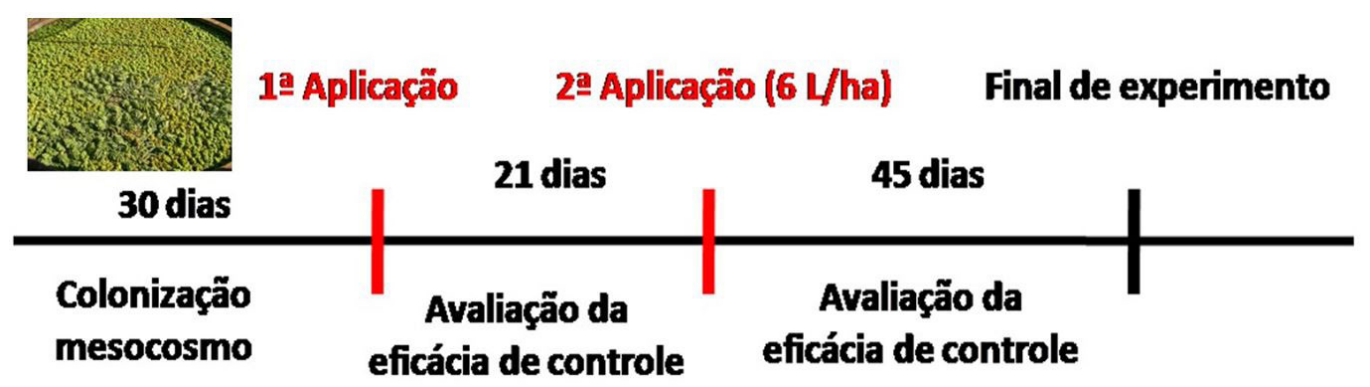

Figura 1. Esquema da colonização dos mesocosmos e procedimentos de aplicação do glyphosate para o controle das espécies de salvinias. 
os três mesocosmos de controle com as três espécies avaliadas (figura 1).

A pulverização foi realizada com pulverizador costal, a pressão constante de 1,8 bar mantida por $\mathrm{CO}_{2}$, equipado com 4 pontas de aplicação XR $11002 \mathrm{VK}$, com espaçamento entre si de $0,50 \mathrm{~m}$ e altura do mesocosmos de $0,50 \mathrm{~m}$, com volume de calda equivalente a $200 \mathrm{~L} \mathrm{ha}^{-1}$.

Os tratamentos foram dispostos em delineamento inteiramente casualizado (DIC), com 3 repetições (mesocosmos) e 1 controle (testemunha sem herbicida) para cada, todos com as 3 espécies. As avaliações de eficácia de controle foram realizadas por meio de escalas de notas de 0 (zero) a $100 \%$ (SOCIEDADE BRASILEIRA DA CIÊNCIA DAS PLANTAS DANINHAS, 1995). A primeira aplicação foi avaliada aos 3, 7, 15, 21 dias após a primeira aplicação (DAPA) e a segunda, aos 3, $7,15,21,30$ e 45 dias após a segunda aplicação (DASA), tendo o experimento um período total de 66 dias de duração.

\section{Resultados e discussão}

$\mathrm{Na}$ fase inicial do experimento não ocorreu eficácia de controle em nenhuma das doses avaliadas (tabela 1). Aos 15 DAPA, para a S. herzogii, ocorreu $73 \%$ de eficácia controle nas doses de $2.880,0$ e $3.360,0 \mathrm{~g} \mathrm{ha}^{-1}$ e de $80 \%$ com $3.840,0 \mathrm{~g} \mathrm{ha}^{-1}$. Para a S. molesta, a eficácia de controle variou de $80 \%$ a $85 \%$ e para a S. Oblongifolia, de $90 \%$ a $93 \%$, em todas as doses. Aos 21 DAPA, os resultados foram similares aos observados na avaliação anterior para $S$. herzogii e $S$. molesta (tabela 1), similar, também, para essas duas espécies de salvinias, com a utilização de 3.360,0 $\mathrm{g} \mathrm{ha}^{-1}$ e $3.840,0 \mathrm{~g} \mathrm{ha}^{-1}$ em condição de casa de vegetação (CRUZ et al., 2015a). Para Emerine
(2010), o glyphosate em $2.240,0 \mathrm{~g} \mathrm{ha}^{-1}+0,25 \%$ de Induce ${ }^{\circledR}$ resultou em $89 \%$ de controle de $S$. molesta 5 semanas após a aplicação.

Para S. oblongifolia a eficácia variou de $90 \%$ a $100 \%$, em todas as doses (tabela 1). Entre as espécies avaliadas, S. oblongifolia foi a mais sensível ao glyphosate, provavelmente devido à posição horizontal de suas folhas e ao menor número de tricomas (BARTHLOTT, 2009), o que pode ter facilitado a retenção da calda com o herbicida e, consequentemente, a sua penetração na epiderme foliar.

Depois de 21 dias da primeira aplicação (DAPA) realizou-se a segunda aplicação de glyphosate. Depois de 3 e de 7 dias da segunda aplicação (DASA), a eficácia de controle para as 3 espécies permaneceu similar à descrita em 21 (DAPA) (tabelas 1 e 2). Aos 15 e 21 DASA, a eficácia de controle variou de $85 \%$ a $90 \%$ para S. herzogii e S. molesta e de $98 \%$ a $100 \%$ para S. oblongifolia (tabela 2).

$\mathrm{Na}$ fase final do experimento, aos 45 DASA, ocorreu excelente eficácia para as 3 espécies de salvinias, com $100 \%$ de controle para S. molesta e S. oblongifolia e controle de $90 \%$ a $95 \%$ para S. herzogii (tabela 2). Para a S. Molesta, o glyphosate nas doses de 2.240,0, 4.480,0 e 8.960,0 $\mathrm{g} \mathrm{ha}^{-1}+0,5 \%$ do adjuvante CideKick ${ }^{\mathrm{TM}}$, em 2 volumes de caldas, $935 \mathrm{~L} \mathrm{ha}^{-1}$ e $1.870 \mathrm{~L} \mathrm{ha}^{-1}$, também resultaram em controle de $93,3 \%$ a $97,7 \%\left(935 \mathrm{~L} \mathrm{ha}^{-1}\right)$ e de $95,3 \%$ a $99,1 \%\left(1.870 \mathrm{~L} \mathrm{ha}^{-1}\right)$ (NELSON et al., 2007). A dificuldade de controle de salvinias foi demonstrada com a aplicação de glyphosate com adição de 5 adjuvantes (Kinetic ${ }^{\circledR}$, Optima ${ }^{\circledR}$, LI-700 ${ }^{\circledR}$, MON0818 ${ }^{\circledR}$ e Cygnet $^{\circledR}$ ), sendo que apenas a dose de 22,9 L ha $^{-1}+0,25 \%$ de Optima ${ }^{\circledR}$ ocasionou controle total e sem rebrota da $S$. molesta (FAIRCHILD et al.,

Tabela 1. Notas e porcentagens de controle de 3 espécies de Salvinia após a primeira aplicação (DAPA) do herbicida glyphosate.

\begin{tabular}{|c|c|c|c|c|c|}
\hline \multirow{2}{*}{ Planta-alvo } & \multirow{2}{*}{$\left(\right.$ g i.a. ha $\left.{ }^{-1}\right)$} & \multicolumn{4}{|c|}{ Eficácia de controle em DAA (\%) } \\
\hline & & 3 & 7 & 15 & 21 \\
\hline \multirow{3}{*}{ S. herzogii } & $2.880,0$ & $\mathrm{E}(0)$ & $\mathrm{E}(0)$ & $\mathrm{C}(73)$ & $\mathrm{C}(80)$ \\
\hline & $3.360,0$ & $\mathrm{E}(0)$ & $\mathrm{E}(0)$ & C (73) & C (73) \\
\hline & $3.840,0$ & $\mathrm{E}(0)$ & $\mathrm{E}(0)$ & $\mathrm{C}(80)$ & $\mathrm{C}(76)$ \\
\hline \multirow[t]{3}{*}{ S. molesta } & $2.880,0$ & E $(0)$ & E (0) & $\mathrm{C}(80)$ & C (76) \\
\hline & $3.360,0$ & $\mathrm{E}(0)$ & $\mathrm{E}(0)$ & B (85) & B (85) \\
\hline & $3.840,0$ & E (0) & E (0) & $\mathrm{B}(85)$ & $\mathrm{B}(85)$ \\
\hline \multirow[t]{3}{*}{ S. oblongfolia } & $2.880,0$ & $\mathrm{E}(0)$ & E $(0)$ & B (90) & B (90) \\
\hline & $3.360,0$ & $\mathrm{E}(0)$ & $\mathrm{E}(0)$ & A (93) & A (98) \\
\hline & $3.840,0$ & E (0) & E (0) & B (90) & $\mathrm{A}(100)$ \\
\hline
\end{tabular}


Tabela 2. Notas e porcentagens de controle de 3 espécies de Salvinia após a segunda aplicação (DASA) do herbicida glyphosate.

\begin{tabular}{|c|c|c|c|c|c|c|}
\hline \multirow{2}{*}{ Planta-alvo } & \multicolumn{6}{|c|}{ Eficácia de controle em DASA (\%) } \\
\hline & 3 & 7 & 15 & 21 & 30 & 45 \\
\hline \multirow{3}{*}{ S. herzogii } & $\mathrm{C}(80)$ & $\mathrm{C}(80)$ & B (85) & B (85) & B (90) & B (90) \\
\hline & $\mathrm{C}(73)$ & $\mathrm{C}(80)$ & B (85) & $\mathrm{B}(85)$ & $A(95)$ & $A(95)$ \\
\hline & $\mathrm{C}(76)$ & $\mathrm{C}(80)$ & B (85) & B (85) & $\mathrm{A}(95)$ & A (95) \\
\hline \multirow[t]{3}{*}{ S. molesta } & $\mathrm{C}(76)$ & $\mathrm{C}(80)$ & B (90) & B (90) & B (90) & $\mathrm{A}(100)$ \\
\hline & $\mathrm{B}(85)$ & $\mathrm{B}(85)$ & B (90) & B (90) & B (90) & $\mathrm{A}(100)$ \\
\hline & $\mathrm{B}(85)$ & $\mathrm{B}(85)$ & B (90) & B (90) & $\mathrm{B}(90)$ & $\mathrm{A}(100)$ \\
\hline \multirow[t]{3}{*}{ S. oblongifolia } & $\mathrm{B}(90)$ & $\mathrm{B}(90)$ & $\mathrm{A}(98)$ & $\mathrm{A}(98)$ & $\mathrm{A}(100)$ & $\mathrm{A}(100)$ \\
\hline & A (98) & A (98) & $\mathrm{A}(100)$ & A (98) & $\mathrm{A}(100)$ & $\mathrm{A}(100)$ \\
\hline & $\mathrm{A}(100)$ & $\mathrm{A}(100)$ & $\mathrm{A}(100)$ & $\mathrm{A}(100)$ & $\mathrm{A}(100)$ & $\mathrm{A}(100)$ \\
\hline
\end{tabular}

2002). Para $S$. auriculata ocorreu apenas $82,5 \%$ de controle, aos 60 DAA, com a pulverização da mistura de $75 \mathrm{~mL}$ carfentrazone-ethyl $+3,0 \mathrm{~L} \mathrm{ha}^{-1}$ de glyphosate (CARVALHO et al., 2005).

Outros herbicidas foram testados no controle de salvinias. O carfrentrazone-ethyl, nas doses de 0,028 a $0,224 \mathrm{~kg}$ i.a. $\mathrm{ha}^{-1}$, promoveu controle de $90 \%$ a $98,7 \%$ de $S$. molesta a partir de 2 a 28 DAA. Contudo, além de ocorrer rebrota das plantas, esse herbicida possui restrições de uso por interferir na pesca, natação, irrigação e potabilidade da água (GLOMSKI et al., 2006). O imazapyr também resultou em controle de $70 \%$ e $90 \%$ de S. molesta, nas doses de 3,5 $\mathrm{L} \mathrm{ha}^{-1}$ e 4,0 $\mathrm{L} \mathrm{ha}^{-1}$, respectivamente, aos 45 dias após a aplicação (CRUZ et al., 2015b), resultado similar ao obtido para as 3 espécies de salvinias.

O glyphosate tem apresentado excelente controle de plantas aquáticas flutuantes, porém as pteridófitas aquáticas apresentam crescimento rápido e reprodução por esporos aliados a mecanismos morfofuncionais de proteção, o que dificulta o seu controle. A arquitetura foliar e a cobertura de cera hidrofóbica pode formar um filme de ar sobre a folha submergida por até 17 dias (BARTHLOTT, 2009).

\section{Conclusão}

O manejo por meio da aplicação sequencial (21 dias após a primeira) pode ser uma opção para o controle de espécies de Salvinias, cujas colonizações são consideradas de difícil manejo. Por esse motivo, dada a sua elevada eficácia, o herbicida glyphosate com adição do adjuvante Aterbane $^{\circledR}$ em aplicação sequencial pode ser uma ferramenta para o manejo de salvinias nos corpos hídricos.

\section{Agradecimentos}

Este trabalho foi financiado pela Fundação de Amparo à Pesquisa do Estado de São Paulo, Fapesp, na forma de bolsa de pós-doutoramento do primeiro autor, Proc. 2006/50700-5.

\section{REFERENCIAS}

ANDA, A. et al. Regional evapotranspiration from a wetland in Central Europe, in a 16-year period without human intervention. Agricultural and Forest Meteorology, v. 205, n. 1, p. 60-72, 2015.

BARTHLOTT, S. Classification of trichome types within species of the water fern Salvinia, and ontogeny of the egg-beater tricomes. Botany, v. 87, p. $830-836,2009$.

BORIN, M. et al. Evaluation of Phragmites australis (Cav.) Trin. evapotranspiration in Northern and Southern Italy. Ecological Engineering, v. 37, n. 5, p. 721-728, 2011.

CARVALHO, F. T. D. et al. Eficácia do carfentrazoneethyl no controle de plantas aquáticas latifoliadas em caixas-d'água. Planta Daninha, v. 23, n. 2, p. 305-310, 2005.

CRUZ, C. et al. Glyphosate effectiveness in the control of macrophytes under a greenhouse condition. Planta Daninha, v. 33, n. 2, p. 241247a, 2015a.

CRUZ, C. et al. Imazapyr herbicide efficacy on floating macrophyte control and ecotoxicology for non-target organisms. Planta Daninha, v. 33, n. 1, p. 103-108b, 2015 b. 
EMERINE, S. E. Greenhouse response of six aquatic invasive weeds to imazamox. Journal of Aquatic Plant Management, v. 48, p. 105-111, 2010.

FAIRCHILD, J. F. et al. Efficacy of glyphosate and five surfactants for controlling Giant Salvinia. Journal of Aquatic Plant Management, v. 40, p. 53-58, 2002.

GETTYS, L. A. et al. Biology and control of aquatic plant: A best management practices handbook. 3rd ed. Marietta: Aquatic Ecosystem Restoration Foundation, 2014. 82-83 p.

GLOMSKI, L. A. M. Carfentrazone-ethyl for control of Giant Salvinia. Journal of Aquatic Plant Management, v. 44, p. 136-138, 2006.

MADSEN, J. D; WERSAL, R. M. W.; ROBLES, W. Evaluation of six herbicides for control of swamp smartweed [Persicaria hydropiperoides (Michx.) Small] under flooded and moist soil conditions. Journal of Aquatic Plant Management, v. 53 , p. $224-227,2015$.

MUDGE, C. R. et al. Evaluation of foliar herbicide and surfactant combinations for control of giant salvinia at three application timings. Journal of Aquatic Plant Management, v. 54, p. 32-36, 2016.

NELSON, L. S. et al. Effect of glyphosate rate and spray volume on control of giant salvinia. Journal of Aquatic Plant Management, v. 45, p. 58-61, 2007.

SARTAIN, B. T. et al. Evaluation of six herbicides for the control of water primrose (Ludwigia peploides) (Kunth) P.H. Raven spp. glabrescens. Journal of Aquatic Plant Management, v. 53, p. 134-137, 2015.

SOCIEDADE BRASILEIRA DA CIÊNCIA DAS PLANTAS DANINHAS - SBCPD. Procedimentos para instalação, avaliação e análise de experimentos com herbicidas. Londrina: SBCPD, 1995. 42 p. 